\title{
INEDITHOS: UN PROYECTO \\ DE PEDAGOGÍA HOSPITALARIA \\ DEDICADO A LA MEJORA DE LA CALIDAD \\ DE VIDA DE NIÑOS Y JÓVENES \\ CON ENFERMEDADES RARAS A PARTIR \\ DE LA INTERVENCIÓN E INVESTIGACIÓN \\ CON VOLUNTARIADO UNIVERSITARIO
}

\section{INeDITHOs: a Hospital Pedagogy project devoted to improving the quality of life of children and young people with rare diseases from the intervention, and research with university volunteering.}

\author{
Francisca Negre BenNasar \\ Universidad Islas Baleares \\ Correo-e: xisca.negre@uib.es \\ Sebastià Verger Gelabert \\ Universidad Islas Baleares \\ Correo-e: s.verger@uib.es
}

Recepción: 20 de noviembre de 2016

Envío a informantes: 28 de noviembre de 2016

Aceptación definitiva: 2 de febrero de 2017

Resumen: El objetivo del artículo es presentar una experiencia en Pedagogía Hospitalaria organizada desde la Universidad de las Islas Baleares. Se trata del proyecto IneDithos, cuya principal finalidad es trabajar para la mejora de la calidad de vida de niños y jóvenes con enfermedades raras, desarrollando tres líneas de intervención: apoyo psicopedagógico a los afectados y sus familias, investigación para dar respuesta a las necesidades que se van detectando en este ámbito y formación del alumnado universitario que colabora en el proyecto utilizando la metodología de Aprendizaje-Servicio. La larga trayectoria del proyecto que se inició en el año 2003 ha posibilitado la consolidación de las tres intervenciones dando lugar a una asociación sin ánimo de lucro con el mismo nombre. Este resultado se complementa con la creciente implicación de otras asociaciones como ABAIMAR y FEDER, con las que se mantiene una estrecha colaboración. Así mismo cabe anotar el aumento del número de voluntarios, lo que permite ofrecer atención 
INEDITHOS: UN PROYECTO DE PEDAGOGÍA HOSPITALARIA DEDICADO A LA MEJORA DE LA CALIDAD DE VIDA DE NIÑOS Y JÓVENES CON ENFERMEDADES RARAS... FRANCISCA NEGRE BENNASAR Y SEBASTIÀ VERGER GELABERT

a un mayor número de afectados al tiempo que mejorar la calidad de las intervenciones realizadas gracias a la colaboración e implicación de estudiantes y profesores que, mediante la metodología de Aprendizaje-Servicio, realizan actividades y elaboran trabajos de fin de grado y de máster a partir de las necesidades identificadas en las intervenciones del voluntariado. INeDiTHos ha introducido las enfermedades raras en el contexto universitario sensibilizando a gran parte de la Comunidad Educativa.

Palabras clave: Pedagogía Hospitalaria; enfermedades raras; TIC; Aprendizaje-Servicio.

Aвsтract: This paper presents an experience in Hospital Pedagogy organized by the University of the Balearic Islands. This project is called INeDITHOs and its main objective is to work into improve the quality of life of children and youth with Rare Diseases. The project works in three lines of intervention: psycho-pedagogical support to patients and their families, research to respond to the needs that are detected in this area and the training of university students who collaborates in the project, using the Service Learning methodology. The long trajectory of the project that began in 2003 has made it possible to consolidate the three interventions resulting in a non-profit association with the same name. This result is complemented by the growing involvement of other Associations such as ABAIMAR and FEDER with which close collaboration is maintained. It is also worth noting the increase in the number of volunteers, which allows to offer attention to a higher number of affected while improving the quality of the interventions made thanks to the collaboration and involvement of students and teachers who, through the methodology of Learning and Service, carry out activities and elaborate end-of-degree and master's work based on the needs identified in the volunteer interventions. INeDiTHOs has introduced Rare Diseases in the university context sensitizing a large part of the Educational Community.

KeY wORDs: Hospital Pedagogy; Minority Diseases; ICT; Learning-service.

\section{Introducción}

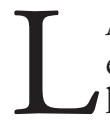

a Organización Mundial de la Salud (oms) afirma que existen unas 7.000 enfermedades raras (ER) que afectan las capacidades físicas, mentales y sensoriales de quienes las padecen (Fernández y Grau, 20I4). En España se estima que existen más de 3 millones de personas afectadas y en la Unión Europea entre 27 y 36 millones, equivalente a un 6-8\% de la población (Eurobarómetro, 20II).

Una ER supone, por lo general, padecer desde el nacimiento o desde muy corta edad una enfermedad crónica, severa y progresiva que puede provocar una discapacidad sensorial, motora o intelectual, para la que no se suele disponer a corto plazo de ningún tratamiento curativo y de la que con demasiada frecuencia resulta complicado obtener información (FEDER, 2009a; FEDER, 2009b).

El 50\% de estas enfermedades empiezan a manifestarse en la primera infancia y, en este sentido, varios estudios ilustran las repercusiones que tiene la enfermedad en el propio niño y en su proceso de escolarización (Violant, Molina y Pastor, 2ori y Lizasoáin, 2016).

INeDITHOS -Investigación e Intervención para la Inclusión Educativa y Tecnológica en Pedagogía Hospitalaria- tiene como objetivo principal mejorar la calidad 
INEDITHOS: UN PROYECTO DE PEDAGOGÍA HOSPITALARIA DEDICADO A LA MEJORA

DE LA CALIDAD DE VIDA DE NIÑOS Y JÓVENES CON ENFERMEDADES RARAS... FRANCISCA NEGRE BENNASAR Y SEBASTIÀ VERGER GELABERT

de vida de niños y jóvenes con ER a partir de la colaboración de voluntariado y estudiantes universitarios utilizando la metodología Aprendizaje/Servicio (Aps). En esta misma línea, se pretende consolidar un espacio de colaboración con docentes e investigadores para formar y sensibilizar a la comunidad universitaria, así como abrir líneas de colaboración transdisciplinar para trabajar en la mejora personal y educativa de los niños y jóvenes con ER. En definitiva, potenciar la transferencia de conocimiento a través de la investigación aplicada para la solución de problemas concretos en el ámbito de la Pedagogía Hospitalaria.

Los responsables de ineDithos son los autores del presente artículo, profesores del Departamento de Pedagogía Aplicada y Psicología de la Educación de la Universidad de las Islas Baleares (UIB). Actualmente colaboran un total de 53 voluntarios: 27 dedicados a la atención en la Unidad de Crónicos y is dedicados a apoyo domiciliario. Además, una parte del voluntariado ejerce doble función dedicándose a otras tareas como mantener y administrar las redes sociales (de momento, Facebook y Twitter), diseñar contenidos gráficos... Por otra parte, se cuenta con 7 profesoras de la Universidad con distintos perfiles (Tecnología Educativa, Educación Inclusiva e Inteligencia Artificial). Es importante anotar que, a medida que las demandas y las necesidades crecen, aumenta el número de personas implicadas en el proyecto.

El inicio de INedithos tuvo lugar en el año 2003 con la finalidad de ofrecer una atención psicopedagógica que mejore la calidad de vida de los pacientes de la Unidad de Crónicos del Hospital Son Espases. Esta Unidad se creó para reubicar a pacientes pediátricos que residían de forma permanente en la UCI hospitalaria. En aquel momento, en dicha Unidad vivían cuatro pacientes de entre 2 y I4 años de edad afectados por diversas enfermedades que les impedían residir en sus domicilios debido a la gravedad de su situación. Así, después de la firma de los convenios pertinentes entre el Hospital y la Universidad y contando únicamente con la colaboración de 4 alumnas

IMAGEN I. Líneas de Intervención de INeDITHOS a partir de 2016

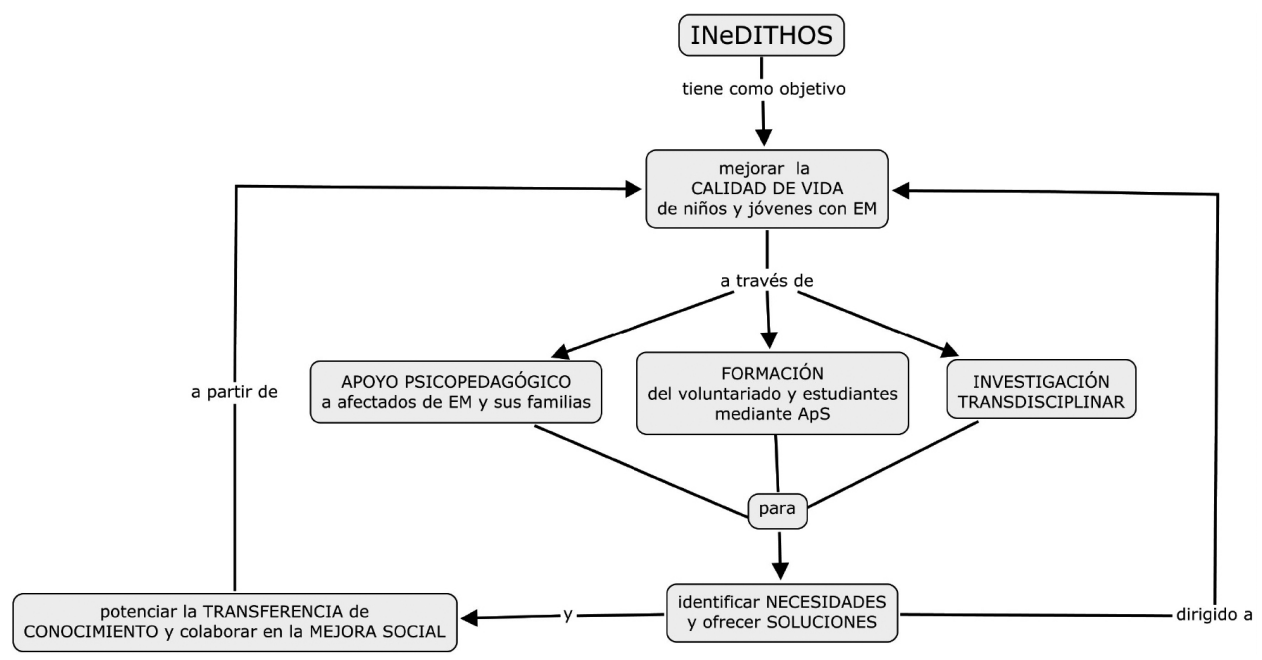

Fuente: Elaboración propia.

(C) EDICIONES UNIVERSIDAD DE SALAMANCA

Aula, 23, 2017, pp. 107-II9

Fuente: Elaboración propia. 
INEDITHOS: UN PROYECTO DE PEDAGOGÍA HOSPITALARIA DEDICADO A LA MEJORA

voluntarias comenzó su intervención. Durante estos años el número de voluntarios ha ido creciendo hasta llegar a las cifras actuales.

Desde su inicio ineDiTHos presenta tres líneas de actuación: el apoyo psicopedagógico a niños y jóvenes con ER y sus familias, la formación del voluntariado y el alumnado universitario a partir de la metodología aps y, finalmente, la investigación dirigida a dar respuesta a las necesidades identificadas a partir de las dos líneas anteriores.

\section{Intervención psicopedagógica en la Unidad de Crónicos y en la Atención Domiciliaria}

La primera actuación consistió en realizar un estudio de necesidades de cada paciente para ofrecer la mejor respuesta a partir de las posibilidades disponibles. Teniendo presentes la edad y las características individuales (tres de los pacientes no tenían alteraciones significativas a nivel cognitivo), se procedió a desarrollar un programa educativo adaptado. Desde el inicio, siempre se ha perseguido buscar un equilibrio entre contenidos académicos y lúdicos con la intención de favorecer al máximo la calidad de vida de los afectados.

De este modo, en el hospital se han desarrollado tres modalidades de trabajo adaptadas a los perfiles de los pacientes con los que se ha trabajado hasta ahora:

- Una línea marcada por el carácter lúdico y de educación en el ocio, dirigido al joven de más edad (actualmente 25 años). Debido a las condiciones personales de este paciente se decidió priorizar desde el principio la socialización y el desarrollo de actividades de ocio. Se trata de un joven que vive en el hospital desde su nacimiento afectado de tetraplejia y, a pesar de no tener ninguna afectación cognitiva, sus características personales suponen un reto para la realización de actividades por parte del voluntariado. Dado que, en la actualidad, los alumnos de la universidad que asisten al hospital tienen una edad muy similar a la suya, las actividades que realizan van dirigidas a compartir gustos musicales, juegos... además de la realización de actividades conjuntas con todos los miembros de la unidad (pacientes, voluntarios y profesionales sanitarios).

- Una segunda línea adaptada a las necesidades de los pacientes más afectados. Con cierta frecuencia en la Unidad de Crónicos se hospitalizan niños y niñas de corta edad en estado de coma. En estos casos, la intervención se centra en un programa de masaje infantil y la narración de cuentos sobre la piel, para posibilitar la interacción del voluntariado considerando la delicada situación del niño e intentando siempre implicar al máximo a la familia.

- Finalmente, contamos con una tercera línea dirigida a ofrecer atención psicopedagógica a dos niñas con las que se empezó a trabajar cuando tenían dos y tres años, respectivamente. Se desarrolló un programa de atención temprana, trabajando principalmente el lenguaje y la estimulación cognitiva. Cuando una de ellas tenía cinco años y tras un largo proceso de reivindicación y trámites burocráticos liderado por la familia, se consiguió que la niña fuera escolarizada en una escuela pública. El curso siguiente también se matriculó en el mismo centro la otra niña. Esta situación, tanto por su complejidad (cada una de ellas necesita una enfermera de la UCI en la escuela durante toda la jornada y una UCI móvil que las recoja y acompañe diariamente del hospital al centro educativo), como por su singularidad 
INEDITHOS: UN PROYECTO DE PEDAGOGÍA HOSPITALARIA DEDICADO A LA MEJORA DE LA CALIDAD DE VIDA DE NIÑOS Y JÓVENES CON ENFERMEDADES RARAS... FRANCISCA NEGRE BENNASAR Y SEBASTIÀ VERGER GELABERT

(no tenemos constancia de otros casos de alumnos que vivan en la UCI hospitalaria y estén escolarizados en una escuela ordinaria) dio un nuevo rumbo a INeDITHOS, ya que desde este momento el apoyo a la escolarización de las niñas se convirtió en un objetivo prioritario. Esto ha supuesto una coordinación regular con tutores y maestros de apoyo del centro y asumir las ayudas necesarias desde el hospital para que puedan seguir su proceso educativo con la máxima fluidez. El objetivo primordial de la escolarización siempre ha sido desarrollar las habilidades sociales y posibilitar una vida lo más normalizada posible. Al cabo de unos años, una de las niñas pudo ir a vivir a su domicilio y en el hospital se continuó trabajando con la otra, quien actualmente está realizando segundo curso de ESO.

Desde el comienzo de nuestra intervención hasta la actualidad, la situación en la Unidad ha ido variando, por lo que ha sido necesario aprender a adaptarnos a un entorno en continuo cambio. Por este motivo, en muchas ocasiones ha sido necesario hacer un replanteamiento en cuanto a metodologías, horarios, actividades, grupos, personal voluntario, e incluso destinatarios, pues durante la intervención ha habido nuevos ingresos que, desafortunadamente, no han podido superar el estado o gravedad en el que se encontraban.

Como se ha comentado, en la Unidad de Crónicos contamos con la colaboración de 27 voluntarios que acuden por grupos todas las tardes de lunes a sábado. Cada sesión tiene un/a responsable de la coordinación de la actividad y del voluntariado.

La gran mayoría de niños y niñas con los que se ha trabajado en el hospital tienen en común que padecen algún tipo de ER, por este motivo trabajamos desde hace años con la Asociación Balear de ER (ABAIMAR) y progresivamente la implicación de INeDITHOS con este colectivo ha ido creciendo y asumiendo nuevos retos. De este modo, desde el mes de enero de 2016 se inició un programa de atención domiciliaria con familias asociadas a dicha entidad. El objetivo principal es ofrecer apoyo psicopedagógico a niños y jóvenes que, por motivos relacionados con su enfermedad, no pueden seguir una escolarización normalizada. Rodríguez y Hernández (20I2: 26) señalan que la atención domiciliaria resulta imprescindible «para prevenir tanto el desfase curricular como el aislamiento social del alumnado convaleciente». En este sentido, se ha considerado el apoyo domiciliario como la mejor manera de paliar, en la medida posible, los efectos de la enfermedad sobre la educación de los alumnos afectados.

Durante el curso 2015/16 se atendieron i2 casos con un total de in voluntarios de la Facultad de Educación y i de la Facultad de Psicología. El proceso de control y valoración de las sesiones se realizaba mediante una hoja de registro. La finalidad era documentar las sesiones para su posterior análisis en vista a valorar el impacto y el grado de satisfacción de los usuarios del servicio. Actualmente, estamos trabajando en esta valoración e incorporando mejoras para la hoja de registro que se ha propuesto para el siguiente curso. Así, en el 20I6/I7 respondemos a I7 demandas, incrementando, por tanto, el número de voluntarios. Algunas de las enfermedades que padecen estos participantes del proyecto son Histiocitosis, Síndrome de coATs, Síndrome de Tourette, Hiperlisinemia recesiva con pipecolemia, Epidermolisis bullosa simple, Enfermedad Mitocondrial, Síndrome de Leigh, Distrofia muscular de Duchenne, Síndrome de Behcet...

En la actualidad INeDiTHOs es un proyecto complejo y ambicioso que planea nuevas propuestas para potenciar la intervención en contextos hospitalarios y domiciliarios, 
INEDITHOS: UN PROYECTO DE PEDAGOGÍA HOSPITALARIA DEDICADO A LA MEJORA

mediante alumnado voluntario y fundamentado en la metodología Aps, complementando acciones solidarias con acciones innovadoras que promueven el aprendizaje activo para el alumnado de la Uiв.

\section{Voluntariado y participación a partir de la metodología Aps}

INeDITHOs ha sido viable gracias a la participación de un grupo de voluntariado que se ha mostrado siempre implicado y motivado para llevar a cabo las propuestas de intervención que han ido surgiendo, debidas, normalmente, a la identificación de nuevas necesidades.

En un principio, el equipo de voluntariado estuvo formado exclusivamente por alumnado de la Facultad de Educación, que sigue siendo mayoritario, pero se ha ampliado con estudiantes de la Facultad de Psicología, Economía, Biología, Derecho, Enfermería, Fisioterapia y otros. Los estudios que aportan mayor número de voluntarios son el grado de Maestro de Educación Primaria, Educación Infantil y Pedagogía. También contamos con la colaboración de alumnado de postgrado, concretamente de los Másteres Universitarios de Educación Inclusiva y Tecnología Educativa: E-learning y Gestión del Conocimiento. Siguiendo a Arevena (2013), valoramos la importancia de racionalizar y sistematizar los cambios en los programas de formación inicial de maestros, por lo que se ha considerado interesante para los estudiantes poner a su disposición nuevas rutas de aprendizaje y presentar propuestas de renovación.

A pesar que desde 2003 INeDithos se ha sostenido gracias al voluntariado, no debemos olvidar, tal como recuerdan Vecina, Chacón y Sueiro (2009), que este tipo de colaboración es un recurso escaso que hay que cuidar convenientemente, implantando «procedimientos eficientes de gestión que maximicen la calidad de los programas, la satisfacción y la permanencia de los voluntarios» (Vecina et al., 2009: II2). Es por todo ello y, fundamentalmente, porque entendemos que la Universidad debe generar modelos de colaboración con diferentes instituciones sociales, por lo que se decide implementar la metodología aps como forma de revalorizar la figura del voluntario. De esta forma se percibe la Universidad como una Institución de Formación Superior responsable de transformar y mejorar la sociedad, en este caso, mediante el apoyo y la atención psicopedagógica de niños y jóvenes con ER, ya sea en la Unidad de Crónicos o en los propios domicilios o, incluso, colaborando en procesos de investigación iniciados por el equipo (fundamentalmente, en forma de trabajo fin de grado-TFG- o trabajo fin de máster-TFM-).

La metodología aps permite concebir las intervenciones realizadas en INeDITHOS como objeto de estudio y colaboración de los estudiantes de la Uiв. Esta metodología se entiende como una forma de aprendizaje inspirada en las pedagogías activas que combina perfectamente con otras metodologías (Batlle, 20I2) y que trata de dar respuesta a problemas reales. Por otra parte, el Aprendizaje Basado en Problemas puede procurar un aprendizaje con mayores cuotas de éxito y se considera un gran aporte en la formación inicial docente, puesto que plantea problemas que ocurren en un contexto educativo y proporciona las bases para enfrentarse a diversas problemáticas (Paineán, 20I2). De esta forma, a partir de la identificación de problemas se inicia una experiencia de Aps. 
INEDITHOS: UN PROYECTO DE PEDAGOGÍA HOSPITALARIA DEDICADO A LA MEJORA DE LA CALIDAD DE VIDA DE NIÑOS Y JÓVENES CON ENFERMEDADES RARAS... FRANCISCA NEGRE BENNASAR Y SEBASTIÀ VERGER GELABERT

Puig y Palos (2006: 6I) define esta metodología como «una propuesta educativa que combina procesos de aprendizaje y de servicio a la comunidad en un solo proyecto bien articulado en el que los participantes se forman al trabajar sobre necesidades reales del entorno con el objetivo de mejorarlo». Entre los beneficios, Jofré y Contreras (2013) indican que favorece la integración, la transferencia y la aplicabilidad del conocimiento.

En este sentido, la metodología aps puede considerarse como una estrategia que permite disponer de contextos reales de aprendizaje en los que se identifican problemas concretos a los que se debe ofrecer una respuesta, permitiendo, por tanto, complementar las competencias y los conocimientos adquiridos durante los estudios universitarios, tanto de grado como de postgrado.

Para Batlle (2009) esta metodología se basa en dos acciones, por una parte, la participación solidaria del voluntariado y, por otra, las posibilidades de aprendizaje que ofrece este tipo de colaboración.

Uno de los objetivos que persigue INediTHos es el de dilucidar los elementos, sus relaciones y las fases de un modelo de colaboración Universidad-Contextos Hospitalarios que brinde líneas de colaboración entre diferentes disciplinas con el objetivo de facilitar la transferencia de conocimiento dirigido a conseguir mejoras sociales. De esta forma, relacionando las competencias de determinadas materias de los diferentes estudios con las necesidades psicoeducativas de los niños y jóvenes con ER se han conseguido diferentes resultados que han significado una primera aproximación para el funcionamiento del proyector. Destacamos, como ejemplo de resultado de un proceso de investigación dirigido a analizar la comunicación Pediatra-Paciente Pediátrico (ver apartado «Investigación en Pedagogía Hospitalaria») y de la aplicación de la metodología Aps, el diseño del Entorno Virtual «Joan y el Asma»² (ver Imagen 2), en el que, juntamente con el equipo de investigación, participaron estudiantes de grado y postgrado.

El objetivo del material multimedia incluido en el entorno es contribuir en la mejora de la calidad de vida del alumnado con enfermedades crónicas (EC), como el asma, que nace a raíz de la ejecución del proyecto Análisis e Intervención Educativa para la mejora de la calidad de vida del alumnado con enfermedades crónicas (EDU2OIO18777), financiado por el Ministerio de Economía y Competitividad.

El material multimedia se dirige a niños y niñas con asma y surgió a partir de las necesidades planteadas trabajando en uno de los objetivos del proyecto: «Análisis de las estrategias didáctico-comunicativas de neumólogos y DUE con pacientes asmáticos».

Para la realización de este material multimedia se contó con la colaboración de diferentes docentes y estudiantes. Destacamos, por ejemplo, la validación de la interfaz de usuario como objetivo del TFG de una estudiante de Pedagogía (Alcover, 20I5) y la validación de los contenidos sobre asma con niños y niñas alumnos de educación primaria en diferentes CEIP de Mallorca como parte del TFM «Educación Inclusiva» (Vila, 20I5). Actualmente se está realizando un segundo TFM, en este caso de «Tecnología Educativa, e-learning y Gestión del Conocimiento», dirigido a valorar la mejora en la autogestión del asma en los niños/as. Se han realizado también otras aportaciones desde la metodología aps en asignaturas de grado (Salinas, 2016a) y asignaturas de

En esta fase de trabajo se consideró interesante centrar el estudio en el asma que, a pesar de no ser una ER, permitió el acceso a una muestra significativa que posibilitó alcanzar resultados relevantes para el estudio de aspectos referidos a las EC y, por tanto, transferibles a las ER.

2 http://joanyelasma.es/proyecto. 
INEDITHOS: UN PROYECTO DE PEDAGOGÍA HOSPITALARIA DEDICADO A LA MEJORA

I I 4 DE LA CALIDAD DE VIDA DE NIÑOS Y JÓVENES CON ENFERMEDADES RARAS...

FRANCISCA NEGRE BENNASAR Y SEBASTIÀ VERGER GELABERT

IMAGEN 2. Diferentes pantallas del material multimedia «Joan y el Asma»
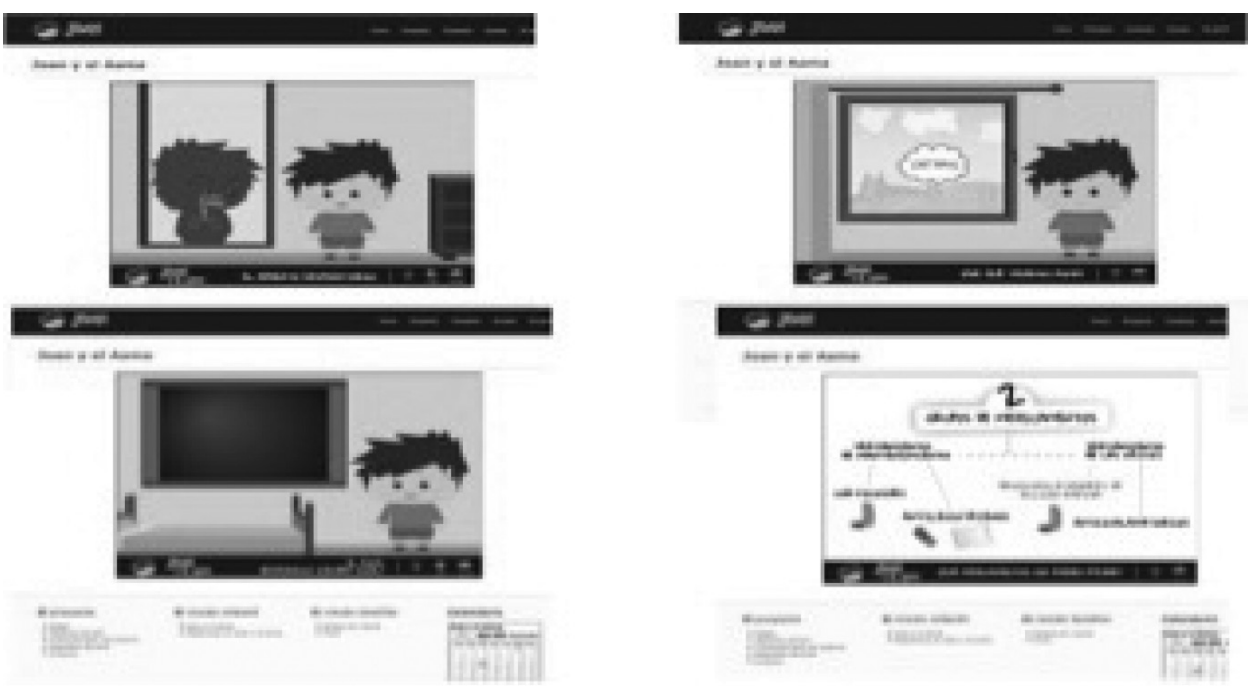

ambos másteres, tesis doctorales (Salom, 20I4), trabajos fin de grado (Salinas, 20I6b) y trabajos fin de máster (Cabanas, 20I7). En la actualidad, se llevan a cabo 3 trabajos fin de grado y 4 trabajos fin de máster.

En general, la valoración de esta metodología es positiva, tanto por parte de los estudiantes como del profesorado implicado y de los usuarios finales y sus familias. Entendemos, pues, que esta metodología complementa la acción del voluntariado a la vez que posibilita nuevas líneas de intervención e investigación en el marco del proyecto INeDITHOS.

\section{Investigación en Pedagogía Hospitalaria}

Tal como hemos señalado, desde los comienzos de INedithos se desarrolla una línea de investigación en el ámbito de la Pedagogía Hospitalaria y, aunque ha ido evolucionando, siempre se ha intentado ofrecer respuestas a las necesidades detectadas. En un principio, los objetivos estaban dirigidos a ofrecer mejoras en la calidad de vida de los pacientes que residían en el hospital y a sus familias, con la intención de desarrollar al máximo sus posibilidades y potenciales.

Las primeras contribuciones se dirigen a la creación de una Comunidad Virtual para facilitar la coordinación entre todos los investigadores. Para ello se realizaron diferentes estudios orientados a identificar las necesidades de INeDITHOs respecto a la coordinación de investigadores de diferentes ramas científicas (Negre, Salinas y De Benito, 2007), el estudio de necesidades integrales de los usuarios de INediTHos (Salinas, Negre y De Benito, 2007) y, por último, un estudio y análisis de herramientas telemáticas para dar respuesta a las necesidades identificadas en los estudios anteriores (De Benito, Negre y Salinas, 2007). La propuesta de Comunidad Virtual se presenta finalmente en Salinas (2008). 
INEDITHOS: UN PROYECTO DE PEDAGOGÍA HOSPITALARIA DEDICADO A LA MEJORA DE LA CALIDAD DE VIDA DE NIÑOS Y JÓVENES CON ENFERMEDADES RARAS... FRANCISCA NEGRE BENNASAR Y SEBASTIÀ VERGER GELABERT

Progresivamente se amplió el interés hacia una visión más global sobre las necesidades que presentan los niños y las niñas con EC y sus familias, tanto desde la perspectiva escolar como en su relación con los profesionales sanitarios. Inicialmente, y a petición del equipo de neumología pediátrica del Hospital Son Espases, se realizó un estudio en el que se analiza el proceso comunicativo que se establece entre el personal sanitario y el paciente asmático pediátrico, con el propósito de determinar cómo mejorar la comunicación entre estos profesionales (pediatras-neumólogos, pediatras-urgencias y personal de enfermería) y los pacientes con asma de edades comprendidas entre los 5 y los I2 años. Concretamente, el estudio se realizó durante I4 meses (junio 2009-septiembre 2010) y se centra en cómo se produjo la aproximación al Servicio de Neumología Pediátrica del Hospital Son Espases, el desarrollo de una propuesta metodológica basada en la observación sistemática en las consultas y habitaciones y el análisis de los resultados obtenidos. Los resultados del estudio (De la Iglesia, Rosselló y Verger, 20I2) sugieren algunas aportaciones con la intención de mejorar el proceso comunicativo, principalmente entre los médicos y los pacientes, tales como: la creación de un glosario de los términos más frecuentes fácilmente comprensible en función de la edad del paciente; explicación detallada de las pruebas diagnósticas que se han de realizar al paciente; explicación detallada del uso de la medicación, especialmente en el caso de los aerosoles; disponer de una batería de materiales gráficos para facilitar la comprensión de la enfermedad que sufre el paciente, etc. A raíz de este trabajo, se realizó conjuntamente entre el equipo de neumólogos y los miembros del proyecto de investigación de INeDiTHos el material multimedia «Joan y el Asma» (Imagen 2).

Posteriormente, se llevó a cabo el proyecto Análisis e intervención educativa para la mejora de la calidad de vida del alumnado con EC (EDU2010/18777), que perseguía un doble objetivo: por una parte, detectar necesidades y propuestas de mejora en los centros educativos que atendían alumnos con alguna enfermedad crónica; y, por otra, detectar, analizar e intervenir sobre las necesidades de carácter educativo que planteaban los profesionales sanitarios (médicos, pediatras y enfermeras), así como los usuarios (pacientes crónicos y familias) en el Servicio de Pediatría del Hospital Universitario Son Espases.

En relación al primer objetivo, se identificaron los facilitadores y los obstáculos para mejorar la Calidad de Vida del alumnado con enfermedad crónica en la escuela, haciendo una especial mención a la materia de Educación Física (Verger, Rosselló y De la Iglesia, 20I6). Se realizaron 43 entrevistas a pediatras y alumnado con enfermedad crónica y 8 grupos de discusión con profesionales sanitarios, profesorado y familias. Los resultados reflejan la coincidencia de todos los colectivos al entender que la escuela y, sobre todo, el tutor pueden ser facilitadores para el adecuado desarrollo físico y emocional del alumnado con alguna enfermedad. Por otra parte, como obstáculos se señalan la falta de comunicación entre los profesionales, la escasez de recursos y la poca o nula participación en las clases de Educación Física, en las salidas y en las actividades extraescolares. A partir de los resultados obtenidos, se propone dotar de profesionales sanitarios y psicólogos para crear una red de apoyo a este colectivo.

Para alcanzar el segundo objetivo se diseñó un método mixto cuantitativo-cualitativo, donde $5 \mathrm{I}$ pediatras y residentes (7I.8\% del servicio) y 78 profesionales de enfermería (95\% del servicio) contestaron un cuestionario; además, 4I de estos 
profesionales participaron en entrevistas analizadas por medio del análisis de contenido. Los resultados obtenidos, coincidiendo con las aportaciones de otro estudio previo (De la Iglesia et al., 20I2), pusieron de manifiesto la importancia de la familia para una buena autogestión de la enfermedad crónica infantil, siendo por ello importante para el profesional sanitario explicar de forma adecuada y en el momento oportuno el proceso de la enfermedad y cómo afrontarla. Sin embargo, los datos mostraron que el colectivo de enfermeras percibía carencias en los conocimientos de índole psicopedagógica relacionadas con el desarrollo de la personalidad y el momento psicoevolutivo del niño y del adolescente, así como con el estado emocional de las familias. En este sentido, los pediatras también expresan los escasos conocimientos sobre algunos aspectos del desarrollo emocional en niños y adolescentes. Por otra parte, se destaca la escasa comunicación existente entre el personal sanitario y los maestros, así como la deficiente información sobre los servicios socioeducativos que se ofrecen, tanto en el hospital, como en la propia comunidad.

En la investigación se llegó a la conclusión de que la pediatría hospitalaria requiere que se tomen en consideración las necesidades psicoemocionales de la familia y del menor, para lo que resulta imprescindible una adecuada formación psicopedagógica, así como el trabajo interprofesional entre sanitarios y educadores. Otro tipo de carencias detectadas se refieren al escaso conocimiento del trabajo que se lleva a cabo en las asociaciones y servicios comunitarios o en los centros escolares. Finalmente, se evidenció la necesidad de implementar estrategias de formación y de trabajo interdisciplinario entre profesionales de la salud, educadores y familias. Además, se ofrecieron pistas para incorporar al proceso formador de los profesionales sanitarios nuevas formas de concebir la educación, acordes con una concepción amplia de la salud, que va más allá de las bases biológicas y de la sintomatología clásica (Rosselló, De la Iglesia, Paz-Lourido y Verger, 2015 y Verger, Paz-Lourido, Rosselló y De la Iglesia, 2015).

\section{Resultados y conclusiones}

INeDiTHOs desarrolla un trabajo desde una perspectiva diferente a la que tradicionalmente se ha venido realizando en la Pedagogía Hospitalaria, en general muy vinculada a las aulas hospitalarias. Tal como hemos descrito, es un proyecto independiente de la administración educativa de las Islas Baleares y muy ligado a las asociaciones de personas enfermas, especialmente ABAIMAR y FEDER. En este sentido, cabe señalar que la evolución realizada por inediTHos ha provocado que en la actualidad se haya convertido en una asociación sin ánimo de lucro, fundamentalmente porque progresivamente existe una mayor demanda social a la que se ha de dar respuesta y ello exige el acceso a determinadas ayudas económicas que aseguren la supervivencia del proyecto y que pueda dar respuesta a las peticiones de intervención que van llegando.

A nuestro entender, la singularidad de inediTHos se basa en que es un proyecto surgido del ámbito universitario que inicialmente pretendía ofrecer una respuesta educativa contextualizada en la UCI de enfermos crónicos del hospital y en los propios domicilios. Ha sido precisamente el contexto universitario el que ha facilitado que se desarrollen las otras líneas del proyecto: por una parte, la formación del 
INEDITHOS: UN PROYECTO DE PEDAGOGÍA HOSPITALARIA DEDICADO A LA MEJORA

DE LA CALIDAD DE VIDA DE NIÑOS Y JÓVENES CON ENFERMEDADES RARAS... FRANCISCA NEGRE BENNASAR Y SEBASTIÀ VERGER GELABERT

voluntariado participante y la aplicación de la metodología aps y, por otra, la investigación en Pedagogía Hospitalaria no solo como objetivo de I+D y proyectos de investigación financiados y dirigidos por personal docente e investigador, también a partir de la colaboración de los estudiantes que deciden realizar sus trabajos fin de grado y fin de máster a partir de las necesidades identificadas durante la trayectoria de ineDithos.

Es difícil augurar hacia dónde y de qué forma evolucionará INeDiTHOs, pero cabe destacar el progresivo aumento de niños y adolescentes atendidos y la óptima respuesta que se está dando entre el alumnado de la universidad, hecho que permite ir creciendo progresivamente. A nivel de investigación, cabe mencionar el proyecto Soluciones educativas para la mejora de la calidad de vida del niño con enfermedad minoritaria desde una intervención innovadora y transdisciplinar (EDU2OI6/79402R) concedido recientemente. Esta investigación persigue analizar la atención educativa y sociosanitaria que recibe el niño y adolescente con ER para diseñar soluciones educativas que mejoren su calidad de vida, mediante la implementación de mecanismos de coordinación y comunicación entre la escuela y los servicios de pediatría y el desarrollo de estrategias inclusivas dirigidas a la sensibilización y la formación de la comunidad educativa.

Para terminar cabe señalar el papel que juega INediTHos como instrumento en la formación y sensibilización de la Comunidad Universitaria respecto a las ER, creando espacios de intercambio y de colaboración dirigidos a la transferencia de conocimiento para la mejora social. La dinámica del proyecto, combinada con la metodología Aps, permite que la gestión del conocimiento generado en la UIB pueda ser compatible en el futuro con aportaciones de otras universidades y/o instituciones.

\section{Bibliografía}

Alcover, A. (2015) Joan y el asma. Diseño y validación de un material didáctico multimedia destinado a pacientes infantiles con asma. Trabajo Fin de Máster. Grado de Pedagogía. Universitat de les Illes Balears.

Aravena, F. (20I3) Desarrollando el modelo colaborativo en la formación docente inicial: la autopercepción del desempeño profesional del practicante en acción. Revista Estudios Pedagógicos, 39 (I), 27-44.

Batlle, R. (2009) Aprendizaje servicio y Educación para la Ciudadanía. Revista vicus. Vigo. Recuperado el is de noviembre de 2016, de http://roserbatlle.files.wordpress.com/2009/o2/ aprendizaje-servicio-y-epc-vicus.pdf.

Batlle, R. (2012) Raons per fer Aprenentatge Servei. Educat, Revista de Psicopedagogia, I2 (marzo y abril 20I2).

Cabanas, R. (20I7) Educación física y enfermedades raras: diseño de una comunidad virtual para docentes. Trabajo Fin de Máster. Universitat Illes Balears.

De Benito, B.; Negre, F. y Salinas, I. (2007) Estudio y análisis de herramientas para la creación de una comunidad virtual de investigación para la atención integral en situaciones de extrema diversidad. En $10 .{ }^{\circ}$ Congreso Iberoamericano EDUTEC 2007. Inclusión digital en la educación superior. Desafios y oportunidades en la sociedad de la información. Buenos Aires, Argentina, 2007.

De la Iglesia, B.; Rosselló, M. R. y Verger, S. (20I2) La relación comunicativa entre el profesional sanitario y el paciente asmático en Pediatría. Revista de Investigación en Educación, I (го), 45-59. 
INEDITHOS: UN PROYECTO DE PEDAGOGÍA HOSPITALARIA DEDICADO A LA MEJORA DE LA CALIDAD DE VIDA DE NIÑOS Y JÓVENES CON ENFERMEDADES RARAS... FRANCISCA NEGRE BENNASAR Y SEBASTIÀ VERGER GELABERT

Eurobarómetro (20ii) Eurobarometer 74.3. Recuperado el io de enero de 20i4, de http:// ec.europa.eu/health/rare_diseases/eurobarometers/index_en.htm.

FEDER (Federación Española de Enfermedades Raras) (2009a) Guía de apoyo psicológico para Enfermedades Raras. Madrid: Autor.

FEDER (2009b) Estudio sobre situación de Necesidades Sociosanitarias de las personas con Enfermedades raras en España. Estudio ENSERio. Madrid: Autor.

FEDER (20IO) Las enfermedades raras. Recuperado el Io de enero de 20I4, de http://www.enfermedades-raras.org/.

Fernández, M. y Grau, C. (20I4) Necesidades educativas, asistenciales y sociales especiales de los niños con enfermedades minoritarias: propuestas para una atención interdisciplinar. Revista de Educación Inclusiva, 7 (3), 97-I24.

Jofre, C. y Contreras, F. (2013) Implementación de la Metodología abP (Aprendizaje Basado en Problemas) en Estudiantes de Primer año de la Carrera de Educación Diferencial. Estudios Pedagógicos [online], 39 (I), 99-II3.

Lizasoáin, O. (2016) Pedagogía hospitalaria: Guía para la atención psicoeducativa del alumno enfermo. Madrid: Síntesis.

Negre, F.; Salinas, I. y De Benito, B. (2007) Comunidades virtuales de investigación y necesidades derivadas de situaciones de extrema diversidad. En $10 .{ }^{\circ}$ Congreso Iberoamericano EDUTEC 2007. Inclusión digital en la educación superior. Desafís y oportunidades en la sociedad de la información. Buenos Aires, Argentina, 2007.

Paineán, O.; Aliaga, V. y Torres, T. (20I2) Aprendizaje basado en problemas: evaluación de una propuesta curricular para la formación inicial docente. Revista Estudios Pedagógicos, 38 (I), I6I-I80.

Puig, J. M. y Palos, J. (2006) Rasgos pedagógicos del aprendizaje-servicio. Cuadernos de Pedagogía, 357, 60-63.

Rodríguez, L. y Hernández, A. M. (2012) Programa de atención domiciliaria: una experiencia práctica. Forum Aragón: Revista Digital de FEAE-Aragón sobre Organización y Gestión Educativa, 4, 24-26. Forum Europeo de Administraciones de Educación-Aragón.

Rosselló, M. R.; De la Iglesia, B.; Paz-Lourido, B. y Verger, S. (20I5) Needs of psychopedagogical training for the care of children with chronic disease: perceptions of hospital nursing. Revista da Escola de Enfermagem da USP, I (49), 37-43.

Salinas, I. (2008) Diseño y validación de un Entorno Virtual para una comunidad de investigadores preexistente. Trabajo Fin de Máster (Máster en Tecnología Educativa: e-learning y gestión del conocimiento). Universitat Rovira i Virgili.

Salinas, I.; Negre, F. y De Benito, B. (2007) Objetivos y necesidades para la creación de una comunidad virtual de investigación para la atención integral en situaciones de extrema diversidad. En $10 .{ }^{\circ}$ Congreso Iberoamericano EDUTEC 2007. Inclusión digital en la educación superior. Desafíos y oportunidades en la sociedad de la información. Buenos Aires, Argentina, 2007.

SAlinas, M. A. (20I6a) Diseño y validación de un material multimedia para trabajar la motivación de un joven con una enfermedad crónica residente en un hospital. En R. RoIG (ed.) Educación y Tecnología. Propuestas desde la investigación y la innovación educativa (pp. 547-548). Alicante: Octaedro.

Salinas, M. A. (2016b) Un país de sonrisas. Diseño de un material multimedia para trabajar la motivación de un joven con una enfermedad crónica residente en un hospital. Trabajo Fin de Máster. Grado de Pedagogía. Universitat de les Illes Balears.

SAlom, M. (2014) Psicopedagogia hospitalària i qualitat de vida: el cas d'Aroa. Tesis doctoral, UIB.

Vecina, M. L.; Chacón, F. y Sueiro, M. J. (2009) Satisfacción en el voluntariado: estructura interna y relación con la permanencia en las organizaciones. Psicothema, 2I (I), II2-II7. 
INEDITHOS: UN PROYECTO DE PEDAGOGÍA HOSPITALARIA DEDICADO A LA MEJORA DE LA CALIDAD DE VIDA DE NIÑOS Y JÓVENES CON ENFERMEDADES RARAS... FRANCISCA NEGRE BENNASAR Y SEBASTIÀ VERGER GELABERT

Verger, S.; Paz-Lourido, B.; Rosselló, M. R. y De la Iglesia, B. (20I5) Necesidades de formación psicopedagógica de pediatras en la atención hospitalaria. Revista de la Facultad de Medicina de la Universidad Nacional de Colombia, 63 (4), 237-245.

Verger, S.; Rosselló, M. R. y De la Iglesia, B. (20I6) Educación Física y atención al alumnado con enfermedad crónica en la escuela. Revista de Psicología del Deporte, 25 (I), 195-200.

VILA, M. (2015) Validación de un material multimedia para la mejora de la comunicación entre el personal sanitario y los pacientes pediátricos con asma. Trabajo Final de Máster. UIB.

Violant, V.; Molina, C. y Pastor, C. (20II) Pedagogía Hospitalaria. Bases para la atención integral. Barcelona: Laertes. 